\title{
A NOTE ON SUBDIRECTLY IRREDUCIBLE DISTRIBUTIVE DOUBLE $p$-ALGEBRAS
}

\author{
M. E. ADAMS and T. KATRIŇÁK \\ (Received 10 Nov 1981, revised 26 Mar 1982 and 10 May 1982) \\ Communicated by J. H. Rubinstein
}

\begin{abstract}
A regular double $p$-algebra $L$ satisfying (i) $\wedge\left(x^{n(+*)} ; n<\omega\right)$ for every $1 \neq x \in L$ and (ii) $L$ is not subdirectly irreducible, is constructed. The construction is purely topological and the desired result is obtained via the known Priestley duality. The notion of an auxiliary regular double $p$-algebra is introduced and the algebras having this property are characterized.

1980 Mathematics subject classification (Amer. Math. Soc.): primary 06 D 15, 06 D 30; secondary 06 E 15,08 B 15.
\end{abstract}

\section{Introduction}

By $\mathrm{H}$. Priestley [9] every distributive (double) $p$-algebra can be associated with a special totally order disconnected compact topological space, and vice versa. By means of this topological duality theory of distributive double $p$-algebras it is possible to dualize every fact and every concept, converting algebraic facts and concepts into topological ones. So, in [4], B. Davey presents a topological characterization of subdirectly irreducible double $p$-algebras. In [7], the same algebras are characterized by algebraic means as follows:

TheOREM A ([7, Theorem 4]). Let $L$ be a distributive double p-algebra and let $|L| \geqslant 3$. Then $L$ is subdirectly irreducible if and only if

\footnotetext{
*The support of the Research Foundation of the State University of New York is gratefully acknowledged by the first author.

(c) Copyright Australian Mathematical Society 1983
} 
(i) $L$ is nearly regular; that is to say, for every $a \in L$,

$$
|G[a]|=\mid\left\{x \in L: x^{*}=a^{*} \text { and } x^{+}=a^{+}\right\} \mid \leqslant 2 ;
$$

(ii) $C(L)=\{0,1\}$;

(iii) If $L$ is regular then there exists $1 \neq d \in D(L)$ such that

$$
x^{n(+*)} \leqslant d
$$

for all $1 \neq x \in D(L)$ and some $n<\omega$;

(iv) If $L$ is not regular then for all $1 \neq x \in D(L)$ with $|G[x]|=1$ there exists $d \in D(L)$ satisfying $|G[d]| \neq 1$ such that

$$
x^{n(+*)} \leqslant d
$$

for some $n<\omega$.

The conditions (i)-(iv) are independent.

Furthermore, in [7], the following is also shown.

TheOREM B ([7, Lemma 7]). Let $L$ be a distributive double p-algebra and let $|L| \geqslant 3$. Then $L$ is subdirectly irreducible if and only if $L$ satisfies the conditions (i), (iii), (iv) from Theorem $\mathrm{A}$ and the condition

(ii') $\wedge\left(x^{n+*)} ; n<\omega\right)=0$ for all $1 \neq x \in L$.

The aim of the present note is twofold. First, it is shown that the conditions of Theorem $\mathrm{B}$ are independent. The crucial example of a regular double $p$-algebra satisfying (i), (ii') and (iv) but not (iii) is constructed by topological means (see Lemmas 1-5). This example also demonstrates the importance of the Priestley's duality. Furthermore, the connections between (ii) of Theorem $A$ and (ii') of Theorem B are investigated. Independently, R. Beazer (see [2], [3]) has considered the interrelationship of conditions similar to those given here. In particular, see [3], he characterized the finitely subdirectly irreducible distributive double $p$-algebras. He then raises the question of whether every finitely subdirectly irreducible regular double $p$-algebra is subdirectly irreducible; we show that this is not the case. Second, the notion of an auxiliary regular double $p$-algebra is introduced. The auxiliary algebras are characterized and, as will be seen, provide another instance of the dual rôle occupied by the conditions (ii) and (ii') of Theorems A and $\mathrm{B}$.

\section{Preliminaries}

A distributive double p-algebra $\left\langle L ; \wedge, \vee,{ }^{*},{ }^{+}, 0,1\right\rangle$ is a bounded distributive lattice $\langle L ; \vee, \wedge, 0,1\rangle$ with two unary operations. For $a \in L, a \wedge x=0$ if and 
only if $x \leqslant a^{*}$ (that is, $a^{*}$ is the pseudocomplement of $a$ ) and, dually, $a \vee x=1$ if and only if $x \geqslant a^{+}$(that is, $a^{+}$is the dual pseudocomplement of $a$ ). Further, $D(L)=\left\{x \in L: x^{*}=0\right\}$ and $C(L)$ is the set of all elements of $L$ that have a complement. In a pseudocomplemented distributive lattice, the relation $x \equiv y(\gamma)$ if and only if $x^{*}=y^{*}$ is a congruence called the Glivenko congruence. Similarly, in a dually pseudocomplemented distributive lattice the dual relation $x \equiv y(\bar{\gamma})$ if and only if $x^{+}=y^{+}$is a congruence. For any distributive double $p$-algebra $x \equiv y(\gamma \wedge \bar{\gamma})$ if and only if $x^{*}=y^{*}$ and $x^{+}=y^{*}$ is a congruence called the determination congruence. Moreover, for $a \in L, a^{\alpha+*)}=a, a^{+*}=\left(a^{+}\right)^{*}$, and for $n \geqslant 0, a^{(n+1)(+*)}=a^{n(+*)+*}$.

An algebra $L$ is finitely subdirectly irreducible if and only if the equality relation is meet irreducible in the congruence lattice $\operatorname{Con}(L)$ of $L$. Observe that, since an algebra is subdirectly irreducible if and only if the equality relation is completely meet irreducible in $\operatorname{Con}(L)$, every subdirectly irreducible algebra is finitely subdirectly irreducible.

For further information on distributive double $p$-algebras, see R. Beazer [1], B. A. Davey [4] or [7].

Since one of the constructions presented here is better described by an ordered topological space, we briefly describe the topological duality for distributive double $p$-algebras as given in $\mathrm{H}$. A. Priestley [9]. A topological space $T$ with a partial ordering is totally order disconnected if and only if, for $x, y \in T$, whenever $x \neq y$ there exists a clopen order-ideal $X \subseteq T$ such that $x \in X$ and $y \notin X$. H. A. Priestley established a duality between the category of bounded distributive lattices and the category of compact totally order disconnected topological spaces. Under this duality, the elements of a bounded distributive lattice correspond to the clopen order-ideals of a compact totally order disconnected space. Such a space is the dual space of a distributive double $p$-algebra if and only if $[X]$ is clopen for every clopen order-ideal $X$ and $(X]$ is clopen for every clopen order-filter. For further information, see H. A. Priestley [9] or B. A. Davey [4].

\section{The conditions (ii) and (ii')}

We begin with the construction of a compact totally order disconnected space that will be needed in the proof of Theorem 1 .

Let $C$ denote the Cantor discontinuum set; that is to say, the set of all real numbers $0 \leqslant t \leqslant 1$ which can be written in the form

$$
t=t_{1} / 3+t_{2} / 3^{2}+\cdots+t_{n} / 3^{n}+\cdots \quad \text { where } t_{i} \in\{0,2\} .
$$


It is known (see, for example, J. L. Kelley [8]) that $C$ is a compact and totally disconnected metric space. More precisely, the sets

$$
\begin{array}{ll}
P_{1}=[0,1 / 3] \cap C, & P_{2}=[2 / 3,1] \cap C, \\
P_{3}=[0,1 / 9] \cap C, & P_{4}=[2 / 9,1 / 3] \cap C, \ldots
\end{array}
$$

are clopen and they form a base of the topology on $C$. It is easy to see that $P_{m}=[a, b] \cap C$ if and only if (i) $a_{n}, b_{n} \in\{0,2\}$ for every $n<\omega$, (ii) there exists an integer $k \geqslant 1$ such that $a_{n}=b_{n}$ for all $n \leqslant k$, and (iii) $a_{n}=0$ and $b_{n}=2$ for every $n>k$. If there is no danger of confusion we shall write plainly $[a, b]$ for $[a, b] \cap C$, whenever $a, b \in C$.

For every $n<\omega$ there is a mapping $\tau_{n}: C \rightarrow C$ defined in the following way:

(i) for $x_{n}=2, \tau_{n}(x)=x$,

(ii) for $x_{n}=0,\left(\tau_{n}(x)\right)_{i}=x_{i}$ for every $i<n,\left(\tau_{n}(x)\right)_{n}=2$,

and $\left(\tau_{n}(x)\right)_{i}=x_{i-1}$ for every $i>n$. It can be readily proved that $\tau_{n}$ is a continuous mapping for every $n<\omega$.

For two elements $x, y \in C$ we have $x=y$ if and only if $x_{n}=y_{n}$ for every $n<\omega$. Moreover,

$$
x<y \quad \text { if and only if } x_{i}<y_{i} \text { for the first } i<\omega \text { with } x_{i} \neq y_{i} .
$$

For more details on the set $C$, see J. L. Kelley [8].

Denote by $\bar{C}$ a homeomorphic image of $C$ satisfying $C \cap \bar{C}=\varnothing$. We shall construct a new partially ordered topological space $S=C \cup \bar{C}$. A set $X \subseteq S$ is said to be open in $S$ if $X=X_{1} \cup X_{2}$ such that $X_{1}$ and $X_{2}$ are open in $C$ and $\bar{C}$, respectively. Evidently, $S$ is again compact and totally disconnected. We shall define a partial order on $S$ as follows: Let

$$
\bar{t}=\bar{t}_{1} / 3+\bar{t}_{2} / 3^{2}+\cdots+\bar{t}_{n} / 3^{n}+\cdots \quad \text { with } \bar{t}_{i}=t_{i} \text { for all } i<\omega
$$

denote the corresponding element from $\bar{C}$ associated with $t \in C$ (see (1)). The mapping $t \rightarrow \bar{t}$ is a homeomorphism between $C$ and $\bar{C}$. The partial order $\subseteq{ }^{*}$ on $S$ can be introduced in the following way:

(i) $i \subseteq{ }^{*} t$ for every $t \in C$,

(ii) having $p, t \in C$ we say that $\bar{p} \subseteq{ }^{*} t$ if $p=\tau_{r}(t)$ for some $r$,

(iii) for $t, z \in S$ and $t \neq z, t \subseteq{ }^{*} z$ if and only if it can occur by (i) or (ii).

LEMMA 1. Let $U$ be an open (clopen) set of the partially ordered topological space $S=\left(S ; \subseteq^{*}\right)$. Then $(U]$ and $[U)$ are open (clopen).

Proof. First we shall assume that $U$ is a base set of $C$, that is $U=[a, b]$ with $a_{n}=b_{n}$ for $n \leqslant k$ and $a_{n}=0, b_{n}=2$ for $n>k$. It is easy to check that

$$
(U]=U \cup \bar{U} \cup \bar{U}_{i_{1}} \cup \cdots \cup \bar{U}_{i_{r}},
$$


where $1 \leqslant i_{1}<\cdots<i_{r} \leqslant k$ is the set of all $1 \leqslant j \leqslant k$ with $a_{j}=0$ and $U_{i_{j}}=$ $\left[\tau_{i_{j}}(a), \tau_{i_{j}}(b)\right]$ for every $j=1, \ldots, r$. (Note that $\bar{U}_{j}=[\bar{c}, \bar{d}]$ is the clopen base set corresponding to $U_{j}=[c, d]$.) Therefore $(U]$ is clopen in $S$. Since $P_{1}, \ldots, P_{m}, \ldots ; \bar{P}_{1}, \ldots, \bar{P}_{m}, \ldots$ form a base of $S$ (see (2)), $(U]$ is an open set whenever $U$ is open. For $U$ clopen, there is a finite family of $P_{i_{1}}, \ldots, P_{i}, \bar{P}_{j_{1}}, \ldots, \bar{P}_{j_{s}}$ such that

$$
U=P_{i_{1}} \cup \cdots \cup P_{i_{r}} \cup \bar{P}_{j_{1}} \cup \cdots \cup \bar{P}_{j_{s}},
$$

because $U$ is compact. Hence $(U]$ is also clopen.

For the second part of the proof take first $U=\vec{P}$ for a base set $P=[a, b]$ with $a$ and $b$ as above (see also (2)). Evidently, for every $c \in C$ with $c_{r}=2$ and $c_{r+1}=0$ there is a unique $c \neq d \in C$ with $\tau_{r}(d)=c$, that is, $d=\tau_{r}^{-1}(c)$. Now, two cases can arise:

(i) $a_{k}=b_{k}=0$ or

(ii) $a_{k}=b_{k}=2$.

In the first event we obtain

$$
[U)=P \cup \bar{P} \cup P_{j_{1}} \cup \cdots \cup P_{j_{s}}
$$

where $1 \leqslant j_{1}<\cdots<j_{s}<k$ is the set of all $1 \leqslant i<k$ with $a_{i}=2, a_{i+1}=0$ and $P_{j_{i}}=\left[\tau_{j_{i}}^{-1}(a), \tau_{j_{i}}^{-1}(b)\right]$ for every $i=1, \ldots, s$. Similarly in the second case

$$
[U)=P \cup \bar{P} \cup P_{j_{1}} \cup \cdots \cup P_{j_{s}} \cup P_{k},
$$

where $P_{j_{i}}$ for $i=1, \ldots, s$ are the same as in the first case and $P_{k}=\left[\tau_{k}^{-1}(a), \tau_{k}^{-1}\left(b^{\prime}\right)\right]$ defining $b^{\prime}{ }_{k+1}=0$ and $b_{n}^{\prime}=b_{n}$ for $n \neq k+1$. Therefore $[U]$ is clopen. The rest of the proof can be left to the reader.

LEMMA 2. The partially ordered topological space $S=\left(S ; \subseteq^{*}\right)$ is compact and totally order-disconnected.

Proof. We have only to prove that for all $x, y \in S$ with $x q^{*} y$ there exists a clopen order-ideal $U$ of $S$ with $y \in U$ and $x \neq U$. Two cases can arise:

(i) $x, y \in C$ or $x, y \in \bar{C}$ or $x \in C$ and $y \in \bar{C}$,

(ii) $x \in \bar{C}$ and $y \in C$.

In the first event we can set $U$ as follows: $U=V \cup \bar{C}$ for $x, y \in C$, where $V$ is a clopen subset of $C$ satisfying $y \in V$ and $x \notin V ; U=V$, where $V$ is a clopen subset of $\bar{C}$ satisfying $y \in V$ and $x \notin V ; U=\bar{C}$.

In the second case we can assume $\bar{x} \in \bar{C}, y \in C$ and $\vec{x} q^{*} y$. Again two cases can occur: $x<y$ or $y<x$.

For $x<y$ there exists a clopen set $P$ from the base of $C$ (see (2)) such that $y \in P$ and $[0, x] \cap P=\varnothing$. By Lemma $l(P]$ is clopen in $S$. Hence, $x<y$ implies $\bar{x} \notin(P]$ and $(P]=U$ is the required set. 
Suppose now $y<x$. Then there exists $k$ such that $y_{n}=x_{n}$ for every $n<k$ and $y_{k}<x_{k}$, that is $y_{k}=0$ and $x_{k}=2$. Since $\bar{x} Z^{*} y$, we have $t=\tau_{k}(y) \neq x$. There exists again $r$ such that $t_{r} \neq x_{r}$ and $t_{n}=x_{n}$ for every $n<r$. Evidently $r>k$. Now, take a clopen set $P=[a, b]$ from (2) defined as follows:

$$
\begin{gathered}
a_{n}=b_{n}=y_{n} \text { for every } n<r, \\
a_{n}=0 \text { and } b_{n}=2 \text { for every } n \geqslant r .
\end{gathered}
$$

Clearly, $y \in P$ and $x \notin P$. By Lemma $1,(P]$ is clopen in $S$. We have only to show that $\bar{x} \notin(P]$. This is equivalent with: $\tau_{n}(p)=x$ for no $p \in P$ and no $n<\omega$. Suppose to the contrary that $\tau_{m}(p)=x$ for some integer $m$ and some $p \in P$. Clearly, $p_{j}=y_{j}$ for every $j \leqslant r-1$ and $p_{m}=0$. Since $p_{k} \neq x_{k}$, we have $m \leqslant k$. But $m<k$ is impossible, as $p_{m}=x_{m}$. The last case $m=k$ is also impossible, because

$$
\left(\tau_{k}(p)\right)_{r}=p_{r-1}=y_{r-1}=\left(\tau_{k}(y)\right)_{r}=t_{r} \neq x_{r},
$$

by hypothesis. Thus $\bar{x} \notin(P]$ and the proof is complete.

A distributive double $p$-algebra is regular if and only if every totally ordered set in its dual space has length $\leqslant 1$. Thus, it follows from Lemmas 1 and 2 that the partially ordered topological space $S=\left(S ; \subseteq^{*}\right)$ is a dual space to a regular double $p$-algebra.

Denote by $C_{2}$ the set of all $x \in C$ satisfying the following property: $x_{n}=2$ for all but finitely many indices $n$. Analogously, the definition of $\bar{C}_{2}$.

Lemma 3. The set $C_{2}$ is dense in $C$.

Proor. Let $x \in C$ and an integer $n$ be given. Take $y=C_{2}$ as follows:

$$
y_{i}=x_{i} \text { for every } i \leqslant n, \quad y_{i}=2 \text { for } i>n .
$$

Therefore $y-x \leqslant 1 / 3^{n}$ and every neighbourhood of $x$ contains an element from $C_{2}$.

LeMmA 4. Let $U \neq S$ be a clopen order-ideal of the ordered topological space $S=\left(S ; \subseteq^{*}\right)$. Then $\wedge\left(U^{n(+*)} ; n \leqslant \omega\right)=\varnothing$.

Proof. It is known that $W^{+}=(S \backslash W]$ and $W^{*}=S \backslash[W)$ for every clopen order-ideal of $S$. Denote by $K=\cap\left(U^{n(+*)} ; n \leqslant \omega\right)$ the set-theoretical intersection of $\left\{U^{n(+*)} ; n<\omega\right\}$. First we prove that $[K) \subseteq K$. Take $d \subseteq{ }^{*} c$ with $d \in K \cap \bar{C}, c \in C$. If $c \in S \backslash U^{n(+*)}$ for some $n$, then $d \in\left(S \backslash U^{n(+*)}\right]$ and consequently $d \notin U^{(n+1)(+*)}$, which is impossible. Thus $c \in K$. $(K] \subseteq K$ is trivial. 
Suppose to the contrary that there exists a clopen order-ideal $V \neq \varnothing$ with $V \subseteq K$. Hence, there exists $P=[a, b] \subseteq V$ from the base (2). We can assume $a_{n}=b_{n}$ for every $n \leqslant k$ and $a_{n}=0, b_{n}=2$ for every $n>k$. We claim that there exists $e \in C$ such that $[0, e] \subseteq V$. Without loss of generality we can assume $a_{k}=b_{k}=0$. Namely, for $a_{k}=b_{k}=2$ take a new element $a<b^{\prime}<b$ defined as follows:

$b_{n}^{\prime}=b_{n}$ for every $n \neq k+1$ and $b_{k+1}^{\prime}=0$. Evidently $\left[a, b^{\prime}\right] \subseteq P, a_{n}=b_{n}^{\prime}$ for $n<k+1, a_{k+1}=b_{k+1}^{\prime}=0$ and $a_{n}=0, b_{n}=2$ for $n>k+1$. Let $a \neq 0$. Define the elements $c, d \in C$ as follows:

Let $m$ be the largest integer with $a_{m}=b_{m}=2$. Clearly, $m<k$. Put

$$
\begin{array}{lll}
c_{n}=d_{n}=a_{n} & \text { for } n<m & \text { and } \\
c_{n}=a_{n+1}, & d_{n}=b_{n+1} & \text { for } n \geqslant m .
\end{array}
$$

Since $x \rightarrow \tau_{m}(x)$ is a homeomorphism between $Q=[c, d]$ and $P$, we see that $Q \subseteq K$. As $Q$ is clopen, we have ( $Q$ ] clopen (Lemma 1) and consequently $(Q] \subseteq V$. Repeating this procedure we obtain finally $[0, e] \subseteq V$.

Let us start with $[0, e] \subseteq V$, where $k \geqslant 2$ is the smallest integer with $e_{n}=2$ for all $n \geqslant k$. We claim that $\left[0, e^{\prime}\right] \subseteq V$ for $e_{k-1}^{\prime}=2$ and $e_{n}^{\prime}=e_{n}$ for all $n \neq k-1$. Really, $\left[0, \tau_{k-1}(e)\right] \subseteq V$, because $x \rightarrow \tau_{k-1}(x)$ is a homeomorphism between $[0, e]$ and $\left[\tau_{k-1}(0), \tau_{k-1}(e)\right],[K),(K] \subseteq K$ and $[0, e] \cup\left[\tau_{k-1}(0), \tau_{k-1}(e)\right]=$ $\left[0, \tau_{k-1}(e)\right]$. Continuing in this way we get a sequence $e^{(n)}$ for $n \geqslant 1$ with

$$
e^{(1)}=e, e^{(2)}=\tau_{k-1}\left(e^{(1)}\right), \ldots, e^{(n+1)}=\tau_{k+n-2}\left(e^{(n)}\right), \ldots .
$$

It is easy to see that $e^{(n)} \in K$ and $e^{(n)} \rightarrow e^{\prime}$ in $C$. Since $K$ is closed, we have $e^{\prime} \in K$. Therefore, $\left[0, e^{\prime}\right] \subseteq K$ and consequently $\left[0, e^{\prime}\right] \subseteq V$ as claimed. Repeating this procedure we obtain $[0,1]=C \subseteq V$, and this implies $S \subseteq V$, a contradiction with $S \neq U \supseteq V$.

LEMMA 5. If $U \neq S$ is a clopen order-ideal of the ordered topological space $S=\left(S ; \subseteq^{*}\right)$ then there exists a clopen order-ideal D of $S$ such that

$$
D^{n(+*)} \nsubseteq U
$$

for all $n<\omega$.

Proof. By hypothesis $I=C \cap(S \backslash U) \neq \varnothing$ and clopen. There exists $b \in I \cap$ $C_{2}$ (Lemma 3). We need two more elements $a^{(1)}, a^{(2)} \in C$ :

$a_{i}^{(1)}=b_{i}$ for $i<k$ and $a_{i}^{(1)}=0$ for $i \geqslant k$, where $k$ is the smallest integer with $b_{i}=2$ for all $i \geqslant k$;

$a^{(2)}=\tau_{k}\left(a^{(1)}\right)$. Two cases can arise: (i) there exists a largest $r$ with $b_{r}=2$ and $b_{r+1}=0$ (in fact $r<k$ ) or (ii) there exists no such $r$. In the first event consider the element $c \in C_{2}$ defined as follows: $c_{i}=b_{i}$ for $i<r, c_{r}=0$ and $c_{i}=2$ for 
$i>r$. Evidently, $c<a^{(1)}<a^{(2)}<b$. Take the clopen set $R=[0, c] \cup\left[a^{(2)}, 1\right]$ in $C$. Therefore $S \neq D=(R]$ is a clopen order-ideal of $S$ (Lemma 1). In the second case we take $S \neq D=\left(\left[a^{(2)}, 1\right]\right]$.

First we make some observations. For an element $e \in C_{2}$ let $l_{0}(e)$ (that is to say, the zero-length of $e$ ) denote the number of all indices $n$ with the property $e_{n}=0$. We claim that

$$
x \in C_{2}, \quad l_{0}(x)=l_{0}(b) \quad \text { implies } x \in D .
$$

Really, let us consider the first event, that is $b_{r}=2$ and $b_{r+1}=0, x \in C_{2}, x \neq b$ and $l_{0}(x)=l_{0}(b)$. Let $j$ be the smallest integer with $x_{j} \neq b_{j}$. If $j<r$ then $x \in D$. If $j=r$ then $x \leqslant c$ and, hence, $x \in D$. Assume $j>r$ and $x_{j}=0$. Hence, $b_{j}=2$. However, $j<k$ is impossible, because $b_{k-1}=0$ and this yields a contradiction with $r<j$. Therefore $j \geqslant k$. This case cannot occur since $l_{0}(x)=l_{0}(b)$. Concluding we have $j>r$ and $x_{j}=2$. Hence $x \geqslant b$ and, consequently, $x \in D$, as claimed. The second case is trivial.

It is easy to check that $x \in C_{2}$ implies $l_{0}(x)=l_{0}\left(\tau_{n}(x)\right)$. Similarly, $\tau_{n}(y)=x$ implies $l_{0}(y)=l_{0}(x)$, that is $l_{0}(x)=l_{0}\left(\tau_{n}^{-1}(x)\right)$. Summarizing, we can say that $D$ contains all elements $x \in C_{2} \cup \bar{C}_{2}$ with the property: $l_{0}(x)=l_{0}(b)$.

We claim that $x \in C_{2} \cup \bar{C}_{2}$ with $l_{0}(x)=l_{0}(b)$ implies $x \in D^{n(+*)}$ for every $n<\omega$. For $n=0$ (recall $D^{\alpha+*)}=D$ ) it is true. Suppose that $D^{n(+*)}$ possesses this property. Therefore, $D^{n(+*)+}=\left(S \backslash D^{n(+*)}\right]$ does not contain elements of zero-length equal to $l_{0}(b)$. The same is also true for $\left[D^{n(+*)+}\right)$. Since

$$
D^{(n+1)(+*)}=S \backslash\left[D^{n(+*)+}\right),
$$

we see that $D^{(n+1)(+*)}$ contains all elements of zero-length $l_{0}(b)$, as claimed. Concluding, we have $b \in D^{n(+*)}$ for all $n<\omega$. Since $b \notin U$, we see that $D^{n(+*)}$ $\nsubseteq U$ for all $n<\omega$ and the proof is complete.

In answer to Beazer's question:

Lemma 6. There exists a regular double p-algebra that is finitely subdirectly irreducible but not subdirectly irreducible.

Proof. Consider the regular double $p$-algebra dual to the topological ordered space $S$ of Lemmas 1-5. By Lemma 4, it is finitely subdirectly irreducible (see R. Beazer [3, Theorem 11]). However, by Lemma 5 , it is not subdirectly irreducible (see Theorem B).

THEOREM 1. The conditions (i), (ii'), (iii) and (iv) from Theorem $\mathrm{B}$ are independent. 
Proof. The five-element chain satisfies (ii'), (iii), (iv) but not (i).

In order to show the independence of (ii') let us consider a subdirectly irreducible regular double $p$-algebra $K$ with $|K| \geqslant 3$. Take the regular double $p$-algebra $L=\mathbf{2} \times K$, where 2 is the two-element chain. Evidently $L$ is regular double $p$-algebra. Take $a=(1,0) \in L$. It is easy to check that $a^{n(+*)}=a \neq 0$ for every $n$. Since $D(L)=\{(1, x) \in L: x \in D(K)\}$, we have

$$
(1, x)^{n(+*)} \leqslant(1, d)
$$

for some $n$ and every $1 \neq x \in D(K)$, where $d \in D(K)$ is the element from $K$ satisfying (iii). Thus $L$ satisfies (i), (ii), and (iv) but not (ii').

The regular double $p$-algebra dual to the topological ordered space $S$ considered in Lemmas 1-5 satisfies (i), (ii') and (iv) but not (iii).

Finally, we demonstrate that condition (iv) is independent of (i), (ii') and (iii). We need the algebraic construction of regular double $p$-algebras from [6]. Let $B$ denote the Boolean algebra of all finite and cofinite subsets of $N$ (that is $\{n$ : $n<\omega\}$ ). Set

$$
\begin{aligned}
& \varphi(a)=\{x \in N: x \in a \text { and } x+1 \in a\}, \\
& \psi(a)=\{x \in N: x \in a \text { or } x-1 \in a\},
\end{aligned}
$$

for every $a \in B$. It is easy to verify that $\varphi: B \rightarrow B$ is a $\{0,1, \wedge\}$-homomorphism, $\psi: B \rightarrow B$ is a $\{0,1, \vee\}$-homomorphism and both satisfy

$$
\psi(\varphi(a)) \leqslant a, \quad \varphi(\psi(a)) \geqslant a
$$

for every $a \in B$. Then $K=\left\{(x, y) \in B^{2}: \varphi(x) \geqslant y\right\}$ is a $\{0,1\}$-sublattice of $B^{2}$. Moreover, $K$ is a regular double $p$-algebra (see [6, Theorem 2]) in which for $t=(x, y) \in K$

$$
t^{*}=\left(x^{\prime}, \varphi\left(x^{\prime}\right)\right) \text { and } t^{+}=\left(\psi\left(y^{\prime}\right), y^{\prime}\right)
$$

is true. First we shall show that $K$ satisfies (ii'). Let us consider $t=(x, y) \in K$. Therefore,

$$
t^{+*}=\left(\left(\psi\left(y^{\prime}\right)\right)^{\prime}, \varphi\left(\left(\psi\left(y^{\prime}\right)\right)^{\prime}\right)\right) .
$$

It is not difficult to see that $\varphi\left(\left(\psi\left(y^{\prime}\right)\right)^{\prime}\right) \subseteq\left(\psi\left(y^{\prime}\right)\right)^{\prime} \subset y$ for every $\varnothing \neq y \neq N$. Therefore, $t^{+*}<t$ for every $0 \neq t \neq 1$. Now it is easy to see that $\wedge\left(t^{n(+*)} ; n<\right.$ $\omega)=0$ for $y$ finite. Assume $y$ cofinite. Let $r$ be the smallest integer such that $n \geqslant r$ implies $n \in y$. Hence $r-1, r \in \psi\left(y^{\prime}\right)$ and consequently $r-1, r \notin\left(\psi\left(y^{\prime}\right)\right)^{\prime}$. Two cases can arise: there exists a largest integer $k$ with $k \in y$ and $k+1 \notin y$ or there is no such integer. In the first event $k+1 \notin\left(\psi\left(y^{\prime}\right)\right)^{\prime}$. Therefore $k \notin \varphi\left(\left(\psi\left(y^{\prime}\right)\right)^{\prime}\right)$. Proceeding in this way one can show in both cases that

$$
t^{n+*)} \leqslant(N \backslash\{0, \ldots, r+n-1\}, N \backslash\{0, \ldots, r+n-1\})
$$

for $n \geqslant k+2$. Therefore $\wedge\left(t^{n(+*)} ; n<\omega\right)=0$ for every $1 \neq t$. 
Now we shall construct a nearly regular double $p$-algebra $L$ with $L /(\gamma \wedge \bar{\gamma}) \cong$ $K$. It is easy to check that $D(K)=\{(N, y) \in K: y \in B\} \cong B$. Let $R$ be an ideal of $K$ generated by $\{(N, y) \in K: y$ finite $\}$. Since

$$
R \cap D(K)=\{(N, y) \in K: y \text { finite }\}
$$

is a prime ideal of $D(K)$, there exists a filter $\bar{R}$ of $K$ such that $(R, \bar{R})$ is an ideal-filter pair of $K$ (see [7, Corollary to Theorem 3]). By [7, Theorem 2] there exists a nearly regular double $p$-algebra $L$ with $L /(\gamma \wedge \bar{\gamma}) \cong K$ such that, for $x \in L,|G[x]|=2$ if and only if $G[x] \in R \cap \bar{R}$. Clearly, $L$ satisfies (i), (ii') and (iii). Take $x=(N, N \backslash\{0\}) \in D(K)$. By induction,

$$
x^{n(+*)}=(N \backslash\{0, \ldots, n\}, N \backslash\{0, \ldots, n\})
$$

in $K$ for every $n$. Therefore $x^{n(+*)} \neq d$ for all $n$ and every $d \in R \cap D(K)$. Thus, (iv) is not satisfied by $L$ and the proof is finished.

LEMMA 7. Let $L$ be a distributive double p-algebra. Let $a=\wedge\left(x^{n(+*)} ; n<\omega\right)$ for some $x \in L$. Then $a \in C(L)$.

Proof. It is well known (see [7]) that

$$
x \geqslant x^{+*} \geqslant \cdots \geqslant x^{n(+*)} \geqslant \cdots \geqslant a .
$$

Therefore $a^{*} \geqslant x^{(n+1)(+*) *}=x^{n(+*)+* *} \geqslant x^{n(+*)+}$ for every $n$. Hence

$$
a^{*+} \leqslant x^{n(+*)++} \leqslant x^{n(+*)}
$$

for every $n<\omega$. Thus, $a^{*+} \leqslant \wedge\left(x^{n(+*)} ; n<\omega\right)$. In a distributive double $p$-algebra we have (see [7])

$$
a \leqslant a^{* *} \leqslant a^{*+} \text { and } a^{*} \leqslant a^{+}
$$

for every element $a$. Therefore $a=a^{*+}$ and $a^{*}$ is a complement of $a$ in $L$, that is $a \in C(L)$.

THEOREM 2. The condition (ii') from Theorem $\mathbf{B}$ implies the condition (ii) from Theorem A. The converse implication is not true.

Proof. Since $a^{n(+*)}=a$ for every $a \in C(L)$, the first statement follows from Lemma 7 and (ii'). The regular double $p$-algebra $L(2 \omega)$ from [7, pages 209, 210] is an example with $C(L(2 \omega))=\{0,1\}$ such that $L(2 \omega)$ does not satisfy the condition (ii'). 


\section{Auxiliary algebras}

Motivated by [7] and the last example in the proof of Theorem 1, we make the following definition.

DEFINITION. A regular double p-algebra $L$ is said to be auxiliary if there exists a subdirectly irreducible distributive double p-algebra $K$ such that $L \cong K /(\gamma \wedge \bar{\gamma})$.

Clearly, by way of example, every subdirectly irreducible regular double $p$-algebra is auxiliary.

The following theorem characterized the auxiliary algebras and, interestingly enough, again exhibits the dual rôle played by (ii) and (ii') of Theorems A and B.

THEOREM 3. A regular double p-algebra $L$ is auxiliary if and only if it satisfies the following conditions:

(i) there exists a prime ideal $P$ of $D(L)$ such that for every $1 \neq x \in D(L)$ there exists $d \in P$ and an integer $n$ with

$$
x^{n(+*)} \leqslant d
$$

(ii) $C(L)=\{0,1\}$ (or (ii') $\wedge\left(x^{n(+*)}: n<\omega\right)=0$ for every $\left.1 \neq x \in L\right)$.

Proof. Let $L$ be auxiliary. Then there exists a subdirectly irreducible distributive double $p$-algebra $K$ with $L \cong K /(\gamma \wedge \bar{\gamma})$. If $K$ is regular, then $L=K$ and there exists $1 \neq d \in D(L)$ satisfying

$$
x^{n(+*)} \leqslant d
$$

for every $1 \neq x \in D(L)$ and some $n$ (see Theorem A). Take $P$ a prime ideal of $D(L)$ with $d \in P$. Then (i), (ii) and (ii') follow from Theorems A and B. Suppose now that $K$ is not regular. Then $K$ is nearly regular. By [7, Lemma 4] there exists an ideal-filter pair $(R, \bar{R})$ on $L$. We can take a prime ideal $P$ of $D(L)$ satisfying $P \supseteq R \cap D(L)$. Since $L \cong K /(\gamma \wedge \bar{\gamma})$, we see by Theorems A and B that (ii) and (ii') are true for $L$. It is known (see [7, Lemma 4]) that $|G[x]| \neq 1$ in $K$ if and only if $x \in R \cap \bar{R}$ in $L$. By hypothesis and Theorem A (or Theorem B) for every $1 \neq x \in D(L) \backslash R$ there exists $d \in R \cap D(L) \subseteq R \cap \bar{R}$ such that $x^{n(+*)} \leqslant d$ for some $n$. This is of course true of $x \in R \cap D(L)$ since, for any $1 \neq x \in L$, $x \geqslant x^{n(+*)}$. Thus the condition (i) holds.

Conversely, let $L$ satisfy (i) and (ii) (or (i) and (ii')). Put $R=(P]$ in $L$. By the Corollary to [7, Theorem 3] there exists an ideal-filter pair $(R, \bar{R})$ of $L$. By [7, Theorem 2] there exists a nearly regular double $p$-algebra $K$ such that $K /(\gamma \wedge$ $\bar{\gamma}) \cong L$. It remains to show that $K$ is subdirectly irreducible. This can be easily done by Theorem A or Theorem B. 
COROllaRY. Let $L$ be a subdirectly irreducible regular double p-algebra. Then there exists a subdirectly irreducible distributive double p-algebra $K$ such that $K \neq L$ and $K /(\gamma \wedge \bar{\gamma}) \cong L$.

Proof. $L$ is auxiliary. There exists a prime ideal $P$ of $D(L)$ satisfying the condition (i) of Theorem 3. As above, there exists an ideal-filter pair $(R, \vec{R})$ of $L$ (see the Corollary to [7, Theorem 3]). By [7, Theorem 2] there exists a proper nearly regular double $p$-algebra $K$ which is the desired algebra.

EXAMPLE. An example of a regular double $p$-algebra that is auxiliary but not subdirectly irreducible is the algebra $L$ dual to the topological ordered space $S=\left(S ; \subseteq^{*}\right)$ from Lemmas $1-5$. By Lemma $4, L$ satisfies (ii) from Theorem 3 . It is easy to see that $D(L)=\{U: U$ is a clopen order-ideal in $S$ and $\bar{C} \subseteq U\}$. Let $a \in C_{0}$, that is, $a_{n}=0$ for all but finitely many indices $n$. Take $R=\{U: U$ is a clopen order-ideal in $S$ and $a \neq U$ \}. Evidently $R$ is a prime ideal of $L$. Put $P=R \cap D(L)$. Clearly, $P$ is a prime ideal of $D(L)$. Now we shall show that for $1 \neq x \in D(L) \backslash P$ there exists $d \in P$ with $x^{n(+*)} \leqslant d$ for some $n$. Take a clopen order-ideal $U$ in $S$ with $a \in U$ and $\bar{C} \in U$. Evidently $U \in D(L) \backslash P$. Assume $U \neq S$. We claim that there exists $n$ such that $a \notin U^{n(+*)}$, that is $U^{n(+*)} \in R$. Suppose to the contrary that $a \in \wedge\left(U^{n(+*)} ; n<\omega\right)$. We shall show that $C_{0} \subseteq$ $\wedge\left(U^{n(+*)} ; n<\omega\right)$. Since $C_{0}$ is dense in $C, U \supseteq U^{+*} \ldots$, and $U^{n(+*)}$ are clopen order-ideals in $S$, we get $U=S$, a contradiction. By a straightforward calculation it can be seen that, for $x \in C$,

$$
x \in \wedge\left(U^{n(+*)} ; n<\omega\right) \quad \text { implies } \quad \tau_{k}(x), \tau_{k}^{-1}(x) \in \wedge\left(U^{n(+*)} ; n<\omega\right) .
$$

Using this fact we show first that $a \in \wedge\left(U^{n(+*)} ; n<\omega\right)$ implies $0 \in \wedge\left(U^{n(+*)}\right.$ : $n<\omega)$. Assume that $k$ is the largest integer with $a_{k}=2$. Therefore $\tau_{k}^{-1}(a) \in C_{0}$, $\left(\tau_{k}^{-1}(a)\right)_{n}=a_{n}$ for $n \neq k$ and $\left(\tau_{k}^{-1}(a)\right)_{k}=0$. Evidently, $\tau_{k}^{-1}(a) \in \wedge\left(U^{n(+*)} ; n<\right.$ $\omega)$. Repeating this procedure we obtain $0 \in \wedge\left(U^{n+*)} ; n<\omega\right)$ by finitely many steps. It is not difficult to show that every $x \in C_{0}$ can be obtained from $0 \in C_{0}$ by superposition of finitely many functions $\tau_{r}$. Namely, let $1 \leqslant i_{1}<\cdots<i_{s}$ be the only indices $j$ with $x_{j}=2$. Then

$$
x=\tau_{i_{s}}\left(\cdots\left(\tau_{i_{1}}(0)\right) \cdots\right) .
$$

Therefore, $C_{0} \subseteq \wedge\left(U^{n(+*)} ; n<\omega\right)$ and the proof is complete. Thus $L$ is auxiliary by Theorem 3 .

Concerning the independence of the conditions in Theorem $3,2 \times 3$ is, for example, a non-trivial example of a regular double $p$-algebra that satisfies (i) but fails to satisfy either (ii) or (ii'). By Theorem 3, $L(2 \omega)$ (see the proof of Theorem 2 ) is an example of a regular double $p$-algebra that satisfies (ii) but does not 
satisfy (i). Furthermore, by Theorem 2, (ii') implies (ii); however, whether (ii') implies (i) is, as yet, unresolved.

Problem. Find a regular double p-algebra $L$ such that, for every $1 \neq x \in L$, $\wedge\left(x^{n(+*)} ; n<\omega\right)=0$ which is not auxiliary.

\section{References}

[1] R. Beazer, 'The determination congruence on double p-algebras', Algebra Universalis 6 (1976), 121-129.

[2] R. Beazer, 'Subdirectly irreducible double Heyting algebras', Algebra Universalis 10 (1980), 220-224.

[3] R. Beazer, 'Finitely subdirectly irreducible algebras with pseudocomplementation', Algebra Universalis 12 (1981), 376-386.

[4] B. A. Davey, 'Subdirectly irreducible distributive double $p$-algebras', Algebra Universalis, 8 (1978), 73-88.

[5] G. Grätzer, General Lattice Theory (Birkhäuser Verlag, Basel, 1978).

[6] T. Katriňák, 'Construction of regular double p-algebras', Bull. Soc. Roy. Sci. Liege 43 (1974), 294-301.

[7] T. Katriňák, 'Subdirectly irreducible distributive double $p$-algebras', Algebra Universalis 10 (1980), 195-219.

[8] J. L. Kelley, General Topology (Van Nostrand Reinhold, New York, 1955).

[9] H. A. Priestley, 'The construction of spaces dual to pseudocomplemented distributive lattices', Quart J. Math. Oxford Ser. (2) 26 (1975), 215-228.

Department of Mathematics

State University of New York

New Paltz, New York

U.S.A.
Katedra algebry a teórie čisel Matematicko-fyzikálna fakulta Univerzity Komenského Bratislava Czechoslovakia 